Research Article

\title{
Inhibition of NF-kB 1 (NF-kBp50) by RNA interference in chicken macrophage HD11 cell line challenged with Salmonella enteritidis
}

\author{
Hsin-I Chiang, Luc R. Berghman and Huaijun Zhou \\ Department of Poultry Science, Texas A\&M University, College Station, TX, USA.
}

\begin{abstract}
The NF-kB pathway plays an important role in regulating the immunity response in animals. In this study, small interfering RNAs (siRNA) were used to specifically inhibit NF-kB 1 expression and to elucidate the role of NF-kB in the signal transduction pathway of the Salmonella challenge in the chicken HD11 cell line. The cells were transfected with either NF-kB 1 siRNA, glyceraldehyde 3-phosphate dehydrogenase siRNA (positive control) or the negative control siRNA for $24 \mathrm{~h}$, followed by Salmonella enteritidis (SE) challenge or non-challenge for $1 \mathrm{~h}$ and $4 \mathrm{~h}$. Eight candidate genes related to the signal pathway of SE challenge were selected to examine the effect of NF-kB 1 inhibition on their expressions by mRNA quantification. The results showed that, with a $36 \%$ inhibition of NF-kB 1 expression, gene expression of both Toll-like receptor (TLR) 4 and interleukin (IL)- 6 was consistently and significantly increased at both 1 $\mathrm{h}$ and $4 \mathrm{~h}$ following SE challenge, whereas the gene expression of MyD88 and IL- $1 \beta$ was increased at $1 \mathrm{~h}$ and $4 \mathrm{~h}$, respectively. These findings suggest a likely inhibitory regulation by NF-kB 1 , and could lay the foundation for studying the gene network of the innate immune response of SE infection in chickens.
\end{abstract}

Key words: RNA interference, NF-kBp50, macrophage, Salmonella, chicken.

Received: September 16, 2008; Accepted: April 23, 2009.

\section{Introduction}

Salmonella enterica serovar Enteritidis (SE) has become one of the most common Salmonella serotypes in many countries (Braden, 2006). Epidemic SE emerges as /constitutes the main source of salmonellosis in humans through the consumption of contaminated poultry or shell eggs. SE can persist in the cecum or ovaries of adult birds for months without triggering clinical signs. Colonizing SE can continue to be excreted in faces (horizontal transmission) or through the yolk (vertical transmission) to contaminate other birds in the flock, as well as poultry products such as meat (after slaughtering) and eggs (Tilquin et al., 2005).

Current control of salmonellosis in poultry is mainly through hygiene measures combined with vaccination programs, although the present vaccines are only partially effective (Wigley et al., 2002). Selection of chickens for genetic resistance to Salmonella infection offers an alternative environment-friendly control measure. Macrophages are critical components of the immune system and play significant roles in both innate and acquired immune responses during SE infection. Through the process of phagocytosis, the macrophage is responsible for the clearance and destruction of both intracellular and extracellular

Send correspondence to Huaijun Zhou. Department of Poultry Science, Texas A\&M University, College Station, TX 77843, USA. E-mail: hjzhou@ poultry.tamu.edu. pathogens (Lavric et al., 2008; Ohl and Miller, 2001; Zhang et al., 2008). Previous studies on chickens have shown that macrophages from a Salmonella-resistant line have greater capability for clearing colonizing Salmonella than macrophages from susceptible lines (Wigley et al., 2002). The different biology of macrophages might be associated with greater and more rapid expression of pro-inflammatory cytokines generated through the NF-kB signal pathway (Wigley et al., 2006).

Although gene expression profiling of chicken macrophages has already been undertaken using an avian macrophage-specific cDNA micro-array with lipopolysaccharide (LPS) stimulation (Bliss et al., 2005), the role of NF-kB in the immune response to SE infection in chickens is still unknown. NF-kB is composed of members of the v-rel reticuloendotheliosis viral oncogene homolog (Rel) family. A total of five NF-kB proteins have already been identified in chickens, namely NF-kB 1 (p50/p105), NF-kB 2 (p100/p52), RelA (p65), RelB and c-Rel. Given that NF-kB is the central regulator of the innate immune response to invasive bacteria, and that it is activated by MyD88-dependent signaling of the TLR pathway (Elewaut et al., 1999; Moynagh, 2005), it is essential to elucidate the role of NF-kB in the TLR pathway in macrophages and to further our knowledge on SE pathogenesis in chickens. To do so, RNA interference (RNAi) technology using chemically synthesized siRNA was applied to specifically inhibit NF-kB expression in a chicken macrophage cell line 
(HD11). Expressions of selected genes including receptors, adaptors and cytokines associated with NF-kB pathway were evaluated, both before and after SE challenge in the siNF-kB 1-treated cells. In the present study, gene expression of several candidate genes was influenced by the NF-kB 1 inhibition.

\section{Material and Methods}

\section{Culture of chicken macrophage HD11 cells}

Chicken macrophage HD11 cells, constituting an established chicken myelomonocytic line transformed by the $m y c$-encoding MC29 virus (Van 1996), were used in this study. The HD11 cells were grown in Dulbecco's Modified Eagle's Medium (Sigma-Aldrich, St. Louis, MO), supplemented with $1 \%$ sodium pyruvate (Sigma-Aldrich, St. Louis, MO), 1\% penicillin/streptomycin (Sigma-Aldrich, St. Louis, MO), 1\% glutamax (Invitrogen, Carlsbad, CA), $5 \%$ chicken serum (Sigma-Aldrich, St. Louis, MO) and 5\% fetal-bovine serum (Atlanta biologicals, Lawrenceville, GA) at $37{ }^{\circ} \mathrm{C}$ in a $5 \% \mathrm{CO}_{2}$ incubator, following routine cell-culture procedures.

\section{siRNA synthesis and transfection}

Messenger RNA sequences of chicken NF-kB 1 (NM_205134) and glyceraldehyde 3-phosphate dehydrogenase (GAPDH, NM_204305.1) were used to design siRNA primers as target genes and positive controls, respectively. For each mRNA sequence, three different siRNA primers were designed for targeting distinct sites in order to compare silencing efficacy. All of the siRNA primers were synthesized and annealed by Ambion (Ambion, Austin, TX) (Table 1). A universal negative control siRNA primer (Ambion, Austin, TX), with no specific targeting to the chicken genome, was used to normalize relative gene inhibition of the target gene. Twenty-four hours before transfection, $450 \mu \mathrm{L}$ HD11 cells were transferred onto a 24-well plate (pre-plating) to reach 50 to $80 \%$ confluence, to then be transfected with either NF-kB 1 siRNA (siNF-kB 1) or GAPDH siRNA (siGAPDH) or a scrambled siRNA negative control (siNC), using the chemical transfection reagent siPORT-Amine (Ambion, Austin, TX). Transfection was undertaken according to the manufacturer's manual, with minor modifications. Briefly, $1.5 \mu \mathrm{L}$ of $10 \mu \mathrm{M}$ annealed siRNA were thoroughly mixed and incubated with $4 \mu \mathrm{L}$ of siPORT-Amine in a volume of $50 \mu \mathrm{L}$ of Opti-mem medium (Invitrogen, Carlsbad, CA) at room temperature for $20 \mathrm{~min}$, this then being added to $450 \mu \mathrm{L}$ of HD11 cell culture with gentle agitation for mixing. The conditions for siRNA transfection were optimized by adjusting different transfection parameters, including cell number $\left(2 \times 10^{5}\right.$, $3 \times 10^{5}$ and $4 \times 10^{5}$ cells $/ \mathrm{mL}$ ), siPORT-Amine concentration $(3,4$, and $5 \mathrm{uL} / 50 \mathrm{uL}$ of total reaction volume of siRNA mixture), siRNA concentration ( $20 \mathrm{nM}, 30 \mathrm{nM}$, and $40 \mathrm{nM}$ ) and incubation time $(12 \mathrm{~h}, 24 \mathrm{~h}, 36 \mathrm{~h}$, and $48 \mathrm{~h})$. After incubation, the total RNA of siRNA- (siNF-kB 1, siGAPDH or siNC) treated cells was extracted for first strand cDNA synthesis, relative gene inhibition being measured by real-time quantitative PCR (qRT-PCR) using SYBR green master mix and the ABI prism 7900HT system (Applied Biosystems, Foster, CA).

\section{SE challenge}

An SE isolate (no. 97-11771, kindly provided by Dr. Kogut at College Station, USDA-ARS) was used in the present study. The SE was maintained in glycerol stocks at $-80^{\circ} \mathrm{C}$, and grown overnight in Luria-Bertani broth at $37^{\circ} \mathrm{C}$ to recover the fresh culture. The recovered $\mathrm{SE}$ was adjusted in sterile PBS to a concentration of $1 \times 10^{9} \mathrm{CFU}$ per milliliter, using spectrophotometric absorbance as previously described (Ferro et al., 2004). Before the challenge, the HD11 culture medium was replaced by an antibiotic-free medium and the cells cultured for $2 \mathrm{~h}$ prior to challenge. The number of siRNA-treated HD1 1 cells was calculated from synchronized duplicates of the siNC transfected group. Both siNF$\mathrm{kB} 1$ and siNC transfected HD11 cells were stimulated with non-opsonized SE at a multiplicity of infection (MOI) of 100 , or with sterilized PBS (non-challenged). The treated culture plate was then centrifuged at $1000 \mathrm{x}$ g for $5 \mathrm{~min}$ to maximize the contact between bacteria and cells, and then incubated at $37^{\circ} \mathrm{C}, 5 \% \mathrm{CO}_{2}$ for $1 \mathrm{~h}$ or $4 \mathrm{~h}$ as previously described (Wigley et al., 2002).

In this study we did not carry out phagocytosis assays to compare mock-transfected and NF-kB transfected cells. However, a previous similar study has demonstrated that there was no appreciable influence on the phagocytic capability of CpG-treated HD11 cells that were SE stimulated at different time points (Xie et al., 2003).

\section{Quantitative RT-PCR}

Total RNA was extracted from the SE-challenged and non-challenged cells with a RNAqueous kit (Ambion, Aus-

Table 1 - List of chemically synthesized small interfering RNAs for specific gene silencing.

\begin{tabular}{lcclc}
\hline Primer name & mRNA target & Sense / Anti-sense & Primer sequence & Accession n. in GenBank \\
\hline siGAPDH & GAPDH & Sense & 5'-GGUGCUGAGUAUGUUGUGGtt-3' & NM_204305 \\
& & Anti-sense & 5'-CCACAACAUACUCAGCACCtg-3' & \\
siNF-kB 1 & NF-kB 1 & Sense & 5'-GGAGAGGAUCCGUAUAUUAtt-3' & NM_205134 \\
& & Anti-sense & 5'-UAAUAUACGGAUCCUCUCCtg-3' & \\
\hline
\end{tabular}


tin, TX), according to manufacturer's instructions. The cDNA was synthesized from equal amounts (300 ng) of total RNA with a random hexamer primer from a Thermoscript RT-PCR system kit (Invitrogen, Carlsbad, CA) according to instructions. The cDNAs from different treatments were quantified by qRT-PCR using the ABI prism 7900HT system (Applied Biosystems, Foster, CA) with software setting at the relative quantification mode. Briefly, the $20 \mu \mathrm{L}$ reaction mixtures contained $10 \mu \mathrm{L}$ of SYBR Green PCR Master Mix (Applied Biosystems, Foster, CA), $0.3 \mu \mathrm{M}$ of each specific oligonucleotide primer (Table 2 ) and $1 \mu \mathrm{L}$ of non-diluted first strand cDNA synthesized from $300 \mathrm{ng}$ of total RNA. The conditions for qRTPCR amplification were set up as: 1 cycle at $95^{\circ} \mathrm{C}$ for $10 \mathrm{~min}, 40$ cycles at $95^{\circ} \mathrm{C}$ for $15 \mathrm{~s}$ and $59^{\circ} \mathrm{C}$ for $1 \mathrm{~min}$. Dissociation curves were obtained for each amplified product at the end of amplification. Each individual sample was run in triplicate and the average critical threshold cycle $(\mathrm{Ct})$ used for data analysis. The $\mathrm{Ct}$ values of target genes were normalized by the $\mathrm{Ct}$ value of internal control (chicken $\beta$-actin gene). Within the group of a same siRNA treatment (siNF-kB 1 or siNC), the normalized $\mathrm{Ct}$ value $(\Delta \mathrm{Ct})$ from SE-challenged HD11 cells was compared to the $\Delta \mathrm{Ct}$ from non-challenged cells, the difference $(\Delta \Delta \mathrm{Ct})$ being transformed into $2^{-\Delta \Delta \mathrm{Ct}}$ value as the estimated fold change of the siNF-kB 1 effect. The relative gene expressions (SE-challenged to non-challenged) were represented by fold change at both $1 \mathrm{~h}$ and $4 \mathrm{~h}$ post-infection, and the comparisons of fold change between siNC and siNF-kB 1 treated HD11 cells were carried out at different time points.

\section{Statistical analysis}

Data analysis was undertaken by means of $\Delta \Delta \mathrm{Ct}$ from six individual data points in two biological replicates, the mutual difference being evaluated by two tailed, paired Student's $t$-tests using the Microsoft ${ }^{\circledR}$ Excel 2003 version (Microsoft Corporation, 2003), $\mathrm{p}<0.05$ being considered significant. Data displayed in the Figures were expressed as the means of fold change \pm standard error from six individual data points.

\section{Results}

\section{RNAi efficacy of siGAPDH and siNF-kB 1 in HD11 cells}

Since transfection efficacy of siRNA delivery varies in different target cell types, and in order to establish

Table 2 - List of primers for quantitative real-time RT-PCR analysis.

\begin{tabular}{|c|c|c|c|c|}
\hline mRNA target & & Primer sequence & Accession $\mathrm{n}$. in GenBank & PCR product size (bp) \\
\hline \multirow[t]{2}{*}{$\beta$-actin } & $\mathrm{F}^{1}$ & 5'-ACGTCTCACTGGATTTCGAGCAGG-3' & NM_205518 & 298 \\
\hline & $\mathrm{R}^{2}$ & 5'-TGCATCCTGTCAGCAATGCCAG-3' & & \\
\hline \multirow[t]{2}{*}{ GAPDH } & $\mathrm{F}$ & 5'-GAGGGTAGTGAAGGCTGCTG-3' & NM_204305 & 113 \\
\hline & $\mathrm{R}$ & 5'-CATCAAAGGTGGAGGAATGG-3' & & \\
\hline \multirow[t]{2}{*}{ NF-kB 1} & $\mathrm{~F}$ & 5'-GAAGGAATCGTACCGGGAACA-3' & NM_205134 & 131 \\
\hline & $\mathrm{R}$ & 5'-CTCAGAGGGCCTTGTGACAGTAA-3' & & \\
\hline \multirow[t]{2}{*}{ IL-1 $\beta$} & $\mathrm{F}$ & 5'-GCTCTACATGTCGTGTGTGATGAG-3' & NM_204524 $4^{3}$ & 80 \\
\hline & $\mathrm{R}$ & 5'-TGTCGATGTCCCGCATGA-3' & & \\
\hline \multirow[t]{2}{*}{ IL-6 } & $\mathrm{F}$ & 5'-AGGACGAGATGTGCAAGAAGTTC-3' & NM_204628 & 78 \\
\hline & $\mathrm{R}$ & 5'-TTGGGCAGGTTGAGGTTGTT-3' & & \\
\hline \multirow[t]{2}{*}{ IL-18 } & $\mathrm{F}$ & 5'-CACTGTTACAAAACCACCGC-3' & NM_204608d ${ }^{4}$ & 213 \\
\hline & $\mathrm{R}$ & 5'-CTTAAAAGCCTTGGAGCTGC-3' & & \\
\hline \multirow[t]{2}{*}{ TL1A } & $\mathrm{F}$ & 5'-CCTGAGTTATTCCAGCAACGCA-3' & NM_001024578 & 292 \\
\hline & $\mathrm{R}$ & 5'-ATCCACCAGCTTGATGTCACTAAC-3' & & \\
\hline \multirow[t]{2}{*}{ TLR-4 } & $\mathrm{F}$ & 5'-TGCACAGGACAGAACATCTCTGGA-3' & NM_001030693 ${ }^{6}$ & 347 \\
\hline & $\mathrm{R}$ & 5'-AGCTCCTGCAGGGTATTCAAGTGT-3' & & \\
\hline \multirow[t]{2}{*}{ TLR-15 } & $\mathrm{F}$ & 5'-TGCTGCCACATTTGGAAGATC-3' & NM_001037835 & 131 \\
\hline & $\mathrm{R}$ & 5'-GATCGGTGCTCCACACAAGTC-3' & & \\
\hline \multirow[t]{2}{*}{ TRAF6 } & $\mathrm{F}$ & 5'-AGTAAATACGAGTGCCCGATCT-3' & CK607050 & 176 \\
\hline & $\mathrm{R}$ & 5'-TTAGCGAAGTTGTCTGGAAAAA-3' & & \\
\hline \multirow[t]{2}{*}{ MyD88 } & $\mathrm{F}$ & 5'-AAGTTGGGCCACGACTACCT-3' & NM_001030962 & 216 \\
\hline & $\mathrm{R}$ & 5'-CTGCTGCTTCCTTCGTAAGT-3' & & \\
\hline
\end{tabular}

${ }^{1}$ Forward primer. ${ }^{2}$ Reverse primer. ${ }^{3}$ From Ferro et al., 2004. ${ }^{4}$ From Sadeyen et al., 2004. ${ }^{5}$ From Takimoto et al., $2005 .{ }^{6}$ From He et al., 2006. 
a protocol for siRNA in the HD11 cell line, a positive control siRNA (chicken siGAPDH) was first used to optimize transfection conditions. Three distinct siGAPDH primers (data not shown) were transfected with different primer concentrations, transfection reagent concentrations and HD1 1 cell concentrations, with varying periods for incubation. The optimal transfection condition was defined as the condition under which siGAPDH could induce the highest inhibition of chicken GAPDH expression. In the present study, the optimized condition were as follows: cell concentration of $2.4 \times 10^{5} / \mathrm{mL}$, siPORTAmine (non-diluted): siRNA $(10 \mu \mathrm{M})$ at 2.6:1 (v/v), with an incubation period of $24 \mathrm{~h}$, in which GAPDH expression was reduced by $45 \%$, when compared to the mock transfected group using negative control siRNA primers (Figure 1). The same transfection conditions were then used for delivering siNF-kB 1 primers, similar efficacy of inhibition ( $36 \%$ ) being observed in the NF-kB1 gene. A cross test was set up for measuring GAPDH expression with a siNF-kB 1 treated sample and NF-kB 1 expression with a siGAPDH treated one. Only small reductions of gene expression ( $8 \%$ for GAPDH and $5 \%$ for NF-kB 1 ) were observed within the cells treated with targetunrelated siRNAs. The siRNA primers with the highest inhibitory efficacy (one out of three from siGAPDH primers and one out of six from siNF-kB 1, data not shown) were selected for silencing target genes in SEchallenged HD11 cells (Table 1).

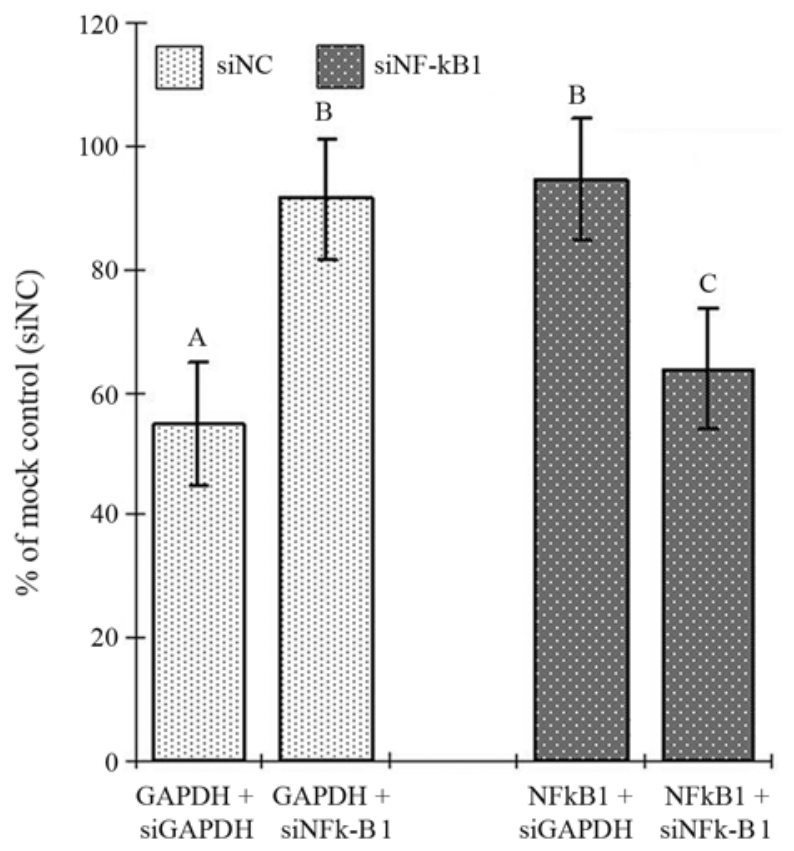

Figure 1 - Reduced mRNA expression (GAPDH and NF-kB 1) in chicken HD11 cells after siGAPDH or siNF-kB 1 treatments. The data are presented as the mean ( \pm standard error) from two replicate experiments. Data with different superscripts are statistically different $(\mathrm{p}<0.05)$.
The effects of reduced NF-kB 1 expression on SE induced immune response

Both siNF-kB 1 treated and negative control (siNC treated) HD 11 cells were followed by SE challenge. The expressions of several candidate genes, including receptors, adaptors, and cytokines, were measured before and after SE challenge ( 1 and $4 \mathrm{~h}$ post-infection). Both TLR 4 and TLR15 were receptor candidate genes due to their critical functions of microbial recognition by binding pathogen-associated molecular patterns (PAMPs). With SE challenge, TLR4 expression was down-regulated at both $1 \mathrm{~h}$ $(-1.75$ - fold) and $4 \mathrm{~h}$ (-3.45 - fold) post-infection (Figure $2 \mathrm{~A})$. Interestingly, the inhibition of siNF-kB 1 was found to significantly increase down-regulated TLR4 gene expression at both time points $(\mathrm{p}<0.05)$. Unlike TLR4, the expression of TLR15 was consistently up-regulated with SE challenge (2.44 - fold at $1 \mathrm{~h}$ and 3.52 - fold at $4 \mathrm{~h}$ postinfection), whereas no significant change was observed in siNF-kB 1 inhibition. Myeloid differentiation primary response gene 88 (MyD88) and TNF receptor-associated factor 6 (TRAF6) genes are important adaptors that assist signal transduction in the canonical TLR pathway. Within siNC treated groups, both MyD88 and TRAF6 were consistently down-regulated with SE challenge $(-2.34$ - fold at $1 \mathrm{~h}$
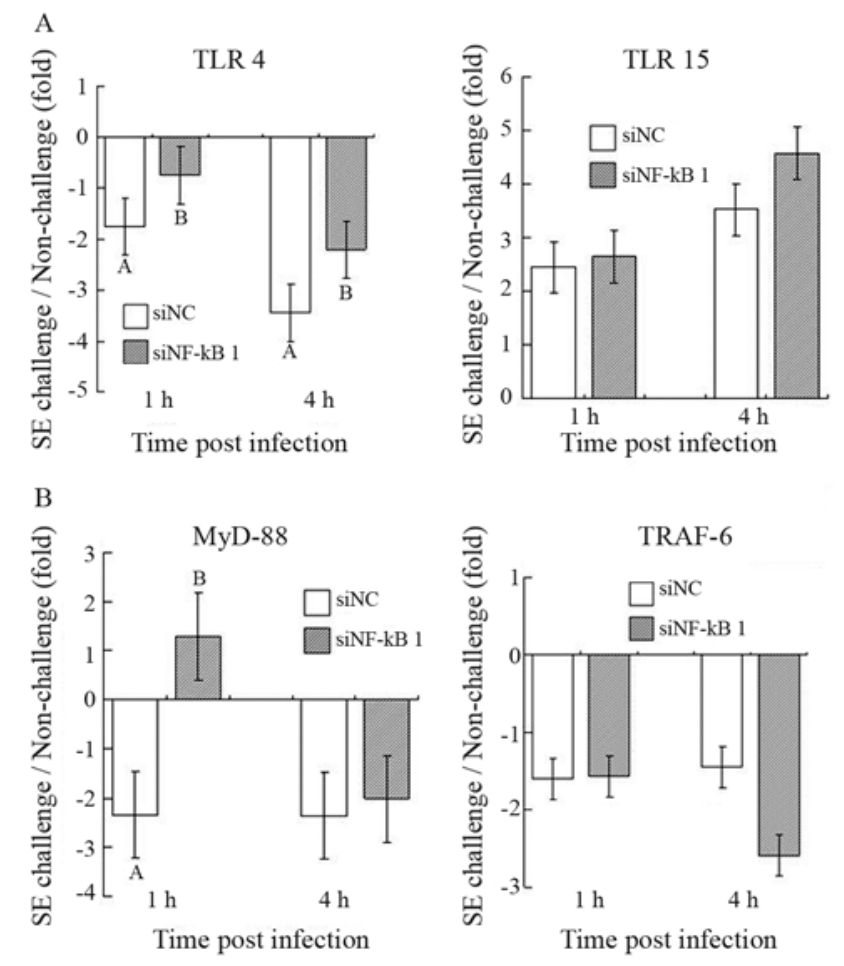

Figure 2 - The effects of NF-kB 1 inhibition on mRNA expression of (A) receptors (TLR4, TLR15) and (B) adaptors (MyD88, TRAF6) from HD11 cells at different points in time, post SE infection. The genes with positive fold changes are up-regulated. The genes with negative fold changes are down-regulated. The data are presented as the mean ( \pm standard error) from two replicate experiments. Data with different superscripts at the same time point are statistically different $(p<0.05)$. 
and -2.37 - fold at $4 \mathrm{~h}$, post-infection for MyD88, and -1.60 - fold at $1 \mathrm{~h}$ and -1.45 - fold at $4 \mathrm{~h}$ post-infection for TRAF6) (Figure 2B). Nevertheless, with NF-kB 1 inhibition, MyD88 was significantly $(\mathrm{p}=0.0047)$ up-regulated 1.28 - fold at $1 \mathrm{~h}$ post-infection, whereas no significant change was found in TRAF6 expression.

Four cytokine genes were chosen to examine the effects of inhibited NF-kB 1 on SE induced immune response. All other cytokine expressions were up regulated after SE challenge except that down regulated expression was observed at $1 \mathrm{~h}$ post-infection in IL-6. Furthermore, gene expressions were consistently higher at $4 \mathrm{~h}$ than $1 \mathrm{~h}$ post-infection (Figure 3). The comparison between siNC and siNF-kB 1 treated samples indicated that IL- $1 \beta$ expression was significantly $(\mathrm{p}=0.001)$ up-regulated by siNF-kB 1 inhibition only at $4 \mathrm{~h}$ post-infection, whereas IL- 6 expression was significantly up-regulated at both $1 \mathrm{~h}$ and $4 \mathrm{~h}$ post-infection ( $p=0.009$ and $p=0.0004$, respectively). No significant effect of siNF-kB 1 inhibition on IL-18 or TL1A was observed.

\section{Discussion}

RNAi is the process of sequence-specific posttranscriptional gene silencing. The mediators of messenger

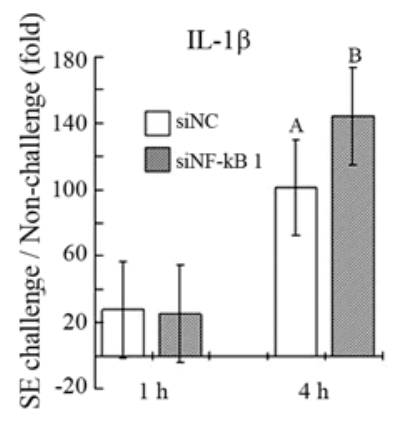

Time post infection

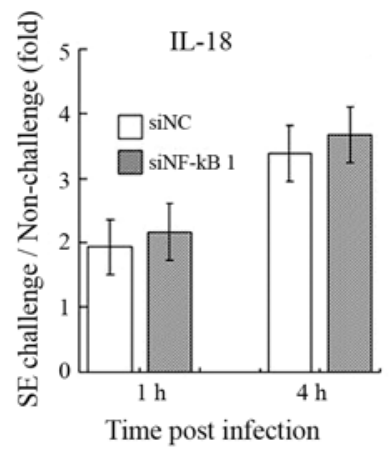

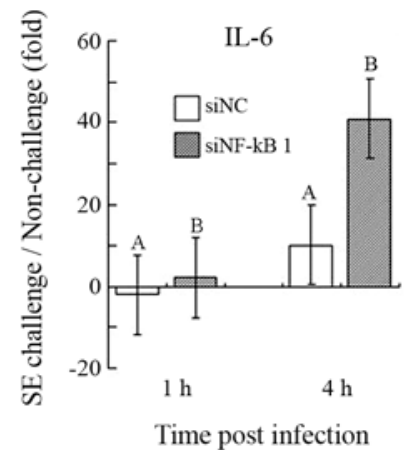

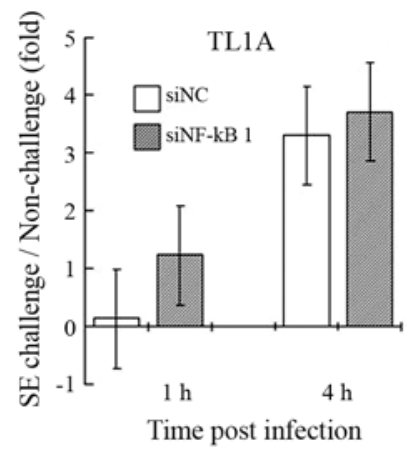

Figure 3 - The effects of NF-kB1 inhibition on mRNA expression of cytokines (IL-1 $\beta$, IL-6, IL-18 and TL1A) in HD11 cells at different time point post SE infection. The genes with positive fold changes are upregulated. The genes with negative fold changes are down-regulated. The data are presented as the mean ( \pm standard error) from two replicate experiments. Data with different superscripts at the same time point are statistically different $(\mathrm{p}<0.05)$.
RNA degradation are 21 26 nucleotide siRNAs generated by a ribonuclease III-like dicer cleaving the longer double strand RNA (dsRNA) (Duxbury and Whang, 2004; Finnegan and Matzke, 2003). RNAi offers great potential for both in vitro target validation and novel therapeutic strategies, based on its mechanism that permits selective inhibition of specific gene expression (Aigner 2006). One of the key experimental approaches to elucidate gene function is to selectively ablate its expression or activity by the loss-of-function (LOF) approach. The gene-knockout approach is not currently applicable in chickens. RNAi using siRNA is more favorable in terms of efficiency, efficacy, and cost compared to other LOF techniques such as antisense DNA oligonucleotides, small molecular inhibitors, and dominant-negative mutants technology (Aigner 2006; Dees et al., 2000; Roth 1986). Along with these features, siRNA has less chance of triggering the interferon response than long double-stranded RNA (Stark et al., 1998). Therefore, siRNA became the first option for LOF selected in the present study.

Most currently reported RNAi experiments in chickens were focused on studying embryogenesis using vector-based RNAi transfection (Chesnutt and Niswander 2004; Das et al., 2006; Kudo and Sutou 2005), and very few studies reported using chemically synthesized short interfering RNAs (siRNAs) on chicken cells (Hu et al., 2002; Sato et al., 2006). The lack of information regarding validated positive and negative control siRNA in chickens makes it more difficult to conduct an effective siRNA experiment using the chicken as a model. Since siRNA gene silence is a transfection-dependent technology, optimization of siRNA transfection was considered the first step to set up a successful siRNA experiment (Duxbury and Whang 2004). We found that the 24-well plate was the most suitable for condition optimization and was capable of providing enough RNA extracted from HD11 cells for mRNA quantification when using qRT-PCR. It has been reported that the efficiency of siRNA transfection is related to intrinsic thermodynamic properties and target site accessibility of the siRNA duplex (Kurreck 2006). In spite of many successful RNAi mediated approaches having been reported, the design of highly potent siRNAs still remains an obstacle. Currently, the approach using a multiple (three or more) primer design targeted to different sites in the target sequence is widely used to overcome this hurdle (Duxbury and Whang, 2004). By using Ambion's design algorithms, our results showed that about $30 \%$ of tested siRNA primers could achieve a reproducible gene knockdown efficacy of around $40 \%$ compared to the siNC groups, which implies that designing three pairs of siRNA primers for one mRNA sequence might be an efficient and economic approach. Although siRNAs are not thought to trigger general translational attenuation through interferon response, as long double-stranded RNA does, the specificity of silencing ability and potential side effects (e.g. transfection reagent 
toxicity) were tested by measuring expression changes in target-unrelated genes. The small reduction in targetunrelated genes in the present study (data not shown) implied that there was no appreciable general translational attenuation, thereby indicating that the observed effects were not biased. In contrast, both target gene and positive control showed a significant reduction in gene expression with the corresponding siRNA treatments. Since the expression of all candidate genes in the SE-challenged cells was measured within $6 \mathrm{~h}$ after NF-kB 1 inhibition, recovery of repressed NF-kB 1 in the present study was unlikely, due to the very short time span allowed for HD 11 cell division.

The macrophage is one of the principle leukocytes involved in defending epithelium cells against Salmonella infection (Ohl and Miller 2001). Macrophages recognize various microbial patterns by many receptors including Fc and complement receptors, integrins, lectins, the mannose receptor, CD14, and the TLRs (Underhill and Ozinsky 2002). One of the most extensively described pathways is the TLR. The predominant signaling pathway used by TLRs results in the activation of NF- $\mathrm{kB}$, a transcription factor that is involved in both T-cell activation and the production of cytokines that promote the extermination of invading microbes (Moynagh, 2005). According to the mammalian model, NF-kB was assumed to participate in signal transduction of the TLR (MyD88- dependent) pathway besides cytokine release in response to bacterial infection (Lynn et al., 2003). However, no direct connection has been confirmed between NF-kB and associated genes involved in the TLR pathway in chickens. In the present study, the role of NF-kB in the TLR pathway was studied by combining NF-kB 1 silencing and SE stimulation of HD11 cells. Eight candidate genes involved in the TLR pathway were analyzed after NF-kB 1 repression. It is assumed that genes with a significant change in expression might have a stronger connection with the NF-kB signal pathway.

Although in chickens LPS can be sensed via the TLR4 receptor, the role of chicken TLR4 in Salmonella infection has been contradictory (Keestra and van Putten 2008; Wigley 2004). Allelic variation in TLR4 was associated with susceptibility to $S$. ser. Typhimurium in chickens (Leveque et al., 2003), whereas Higgs and his colleagues reported that there was no up-regulation of TLR4 in the cecum following $S$. Typhimurium infection (Higgs et al., 2006). Very few studies have reported TLR4 gene expression in response to SE infection in chicken macrophages or HD11 cells. Our results showed down-regulation of TLR4 after SE challenge at both $1 \mathrm{~h}$ and $4 \mathrm{~h}$ post-infection, this being significantly attenuated following the inhibition of NF-kB 1 expression. For TLR15, a novel chicken TLR associated with $S$. Typhimurium infection (Higgs et al., 2006), there was no statistical significance at both $1 \mathrm{~h}$ and 4 $\mathrm{h}$ post-infection, although a slight up-regulation with the inhibition of siNF-kB 1 was observed. The results implied that NF-kB1 might be involved in feedback mediating TLR4 expression through an inhibitory effect, but with no or only minor effects on mediating TLR15 expression.

Based on a MyD88 knock-out mice study (Janssens and Beyaert 2002; O'Neill 2003), it has been found that MyD88 is a universal adaptor for all TIR-domain-containing receptors except TLR-3. After TLRs are activated by their ligand, MyD88 recruits IL-1 receptor-associated kinases (IRAKs) to interact with the TLRs. These activated IRAKs then associate with TRAF6 to activate the IkB kinase (IKK) complex, which finally releases NF-kB by degrading the $\mathrm{IkB} \alpha$ molecule (Takeda and Akira 2004; Yamamoto et al., 2004). Both MyD88 and TRAF6 are downstream adaptors of TLR4, and interestingly, they both showed a consistent down-regulation with SE challenge. However, with the inhibition of siNF-kB 1, a dramatic up-regulation of MyD88 at $1 \mathrm{~h}$ post-infection was observed, while no significant change was found for TRAF6, except for an even lower expression at $4 \mathrm{~h}$ post-infection for some unknown reason. Few studies have reported the detailed regulation of MyD88 or TRAF6 in chickens. In human monotypic THP-1 cells, no significant change of mRNA expression on MyD88 was observed between 0 to $4 \mathrm{~h}$ after LPS stimulation (Tamai et al., 2003). The stimulation of LPS on murine macrophages also showed no significant change in TRAF6 protein levels (Chen et al., 2006). It has been reported that most adaptors function with subtle gene expression in transcriptome during signaling transduction (Ben-Shaul et al., 2005). It is unclear whether the up-regulation of MyD88 in the present study is associated with variant chicken MyD88 isoforms or not (Qiu et al., 2008). However, this is the first study to demonstrate adaptor gene expression change in chicken macrophages as a consequence of SE challenge. Our results also indicate a potential feedback regulation between MyD88 and NF$\mathrm{kB} 1$.

Inflammatory cytokines, such as IL-1 $\beta$, IL-6, IL-12, IL-18, and TNF-alpha, are the end products of the MyD88dependent TLR pathway (Takeda and Akira, 2004; Yamamoto et al., 2004). The production of inflammatory cytokines would be expected in those macrophages where an inflammatory response occurs (Kaiser et al., 2004). In the present study, all cytokines showed significantly upregulated expression at $4 \mathrm{~h}$ post-infection, thereby indicating that SE challenge was sufficient to induce an innate immune response in HD11 cells. More rapid and dramatic expressional fold changes have already been observed for IL-1 $\beta$ (25 to 44 - fold) and IL-6 (-1.9 to 40 - fold), results similar to those reported from a previous study on chicken macrophages with Salmonella challenge (Wigley et al., 2006). Although the synthesis of inflammatory proteins is assumed to be related to NF-kB binding to their cognate promoter, our results showed that repressed NF-kB has significant up-regulatory effects on both IL-1 $\beta$ and IL-6. This was not exactly what we expected. The possible reasons for 
these unexpected results could be: 1) The regulation of pro-inflammatory cytokines by a NF-kB pathway is modulated by the canonical NF-kB heterodimer (p50-p65). The knock-down of one of the components in this heterodimer might not be sufficient to reduce gene expression in these cytokines. This has prompted us to consider including the knock-down of another NF-kB component in our next study; 2) The induction of gene expression in these cytokines might be regulated by another transcription factor, such as AP-1, since AP-1 has been shown to induce cytokine expression along with that of NF-kB (Kogut et al., 2008); 3) It is possible that the binding site of the heterodimer p50-p65 could also be occupied by the homodimer p50-p50, whereupon p50-p50 may function as a repressor to regulate $\mathrm{p} 50$-p 65 's role as a transcription factor essential for immune response (Beinke and Ley, 2004; Tong et al., 2004). In mammals, IL-1 $\beta$ and IL-6 are both critical for activating the immune response and synthesizing acute-phase proteins (Giansanti et al., 2006). It is speculated that these two pro-inflammatory cytokines might be essential in the early phase of the inflammatory stage against Salmonella infection.

The function of IL-18 is associated with enhancing a Th1-type response, besides activating PMN (polymorphonuclear) cells such as neutrophils in mammal (Lee et al., 2004). Swaggerty and her colleagues reported that higher IL-18 mRNA expression in heterophils in chickens (a counterpart of neutrophils in birds) was associated with resistance to SE infection. This suggested that IL-18 may also play a protective role against Salmonella infections (Swaggerty et al., 2006). A previous study in humans reported that repression of IL-18 has weak or absent repression with the competitive inhibition of NF-kB when compared to IL-1 $\beta$ (Lee et al., 2004). In the present study, a rapid increase of IL-18 in chicken macrophages after SE challenge was observed. Notwithstanding, there is no significant effect on IL-18 gene expression with the inhibition of NF-kB 1.

Chicken TL1A (also known as TNF superfamily 15) was suggested to function as a substitute for mammalian TNF- $\alpha$ induced by LPS via the NF-kB pathway (Hong et al., 2006; Takimoto et al., 2005), although very few studies have addressed this novel chicken cytokine. Although no significant effect on TL1A expression was observed after NF-kB 1 repression, our results showed that SE challenge could also induce TL1A expression in chicken macrophages, especially $4 \mathrm{~h}$ post-infection. Increased TL1A may exert an inflammatory function by enhancing nitric oxide (NO) production and heterophil phagocytosis (Takimoto et al., 2005).

Notably, all of the regulatory directions of siNF-kB 1 on cytokines were similar to its effects on TLR genes, supporting the possible function of NF-kB1 as an inhibitory component in the TLR pathway. In mammals, three pathways were reported as activating the NF-kB function through different dimeric complexes (p65-p50, p50-p50 and $\mathrm{p} 52-\mathrm{RelB}$ ). The canonical pathway via p65-p50 heterodimers is essential for an immune response, whereas p50p50 homodimers may function as repressors by competing for the DNA binding site of other NF-kB dimers (Lernbecher et al., 1993). It was also reported that the adenovirus-mediated induced $\mathrm{p} 50$-p50 dimer displayed suppressed IL-1 $\beta$ activation, but had no effect on TNF-alpha in colon-derived HT-29 cells (Tong et al., 2004). The novel findings in the present study have provided new insights into how NF-kB 1 can regulate the TLR signal transduction pathway as a possible inhibitory factor in chickens. Further studies focusing on RelA protein may help to reveal the roles of NF-kB in this sophisticated TLR signal pathway.

\section{References}

Aigner A (2006) Gene silencing through RNA interference (RNAi) in vivo: Strategies based on the direct application of siRNAs. J Biotechnol 124:12-25.

Beinke S and Ley SC (2004) Functions of NF-kappa B1 and NF-kappa B2 in immune cell biology. Biochem J 382:393409.

Ben-Shaul Y, Bergman H and Soreq H (2005) Identifying subtle interrelated changes in functional gene categories using continuous measures of gene expression. Bioinformatics 21:1129-1137.

Bliss TW, Dohms JE, Emara MG and Keeler CL (2005) Gene expression profiling of avian macrophage activation. Vet Immunol Immunopathol 105:289-299.

Braden CR (2006) Salmonella enterica serotype Enteritidis and eggs: A national epidemic in the United States. Clin Infect Dis 43:512-517.

Chen HQ, Wu YF, Zhang YQ, Jin LN, Luo L, Xue B, Lu C, Zhang $\mathrm{XR}$ and Yin ZM (2006) Hsp70 inhibits lipopolysaccharide-induced NF-kappa B activation by interacting with TRAF6 and inhibiting its ubiquitination. FEBS Lett 580:3145-3152.

Chesnutt C and Niswander L (2004) Plasmid-based short-hairpin RNA interference in the chicken embryo. Genesis 39:73-78.

Das RM, Van Hateren NJ, Howell GR, Farrell ER, Bangs FK, Porteous VC, Manning EM, McGrew MJ, Ohyama K, Sacco MA, et al. (2006) A robust system for RNA interference in the chicken using a modified microRNA operon. Dev Biol 294:554-563.

Dees E, Pabon-Pena LM, Goodwin RL and Bader D (2000) Characterization of CMF1 in avian skeletal muscle. Dev Dynam 219:169-181.

Duxbury MS and Whang EE (2004) RNA interference: A practical approach. J Surg Res 117:339-344.

Elewaut D, Didonato JA, Kim JM, O’Neill D, Truong F, Eckmann L and Kagnoff MF (1999) Nuclear factor-kappa B is a central regulator of the intestinal epithelial cell innate immune response induced by infection with enteroinvasive bacteria. J Immunol 163:1457-1466.

Ferro PJ, Swaggerty CL, Kaiser P, Pevzner IY and Kogut MH (2004) Heterophils isolated from chickens resistant to extra-intestinal Salmonella enteritidis infection express higher levels of pro-inflammatory cytokine mRNA following in- 
fection than heterophils from susceptible chickens. Epidemiol Infect 132:1029-1037.

Finnegan EJ and Matzke MA (2003) The small RNA world. J Cell Sci 116:4689-4693.

Giansanti F, Giardi MF and Botti D (2006) Avian cytokines-an overview. Curr Pharm Des 12:3083-3099.

He H, Genovese KJ, Nisbet DJ and Kogut MH (2006) Profile of Toll-like receptor expressions and induction of nitric oxide synthesis by Toll-like receptor agonists in chicken monocytes. Mol Immunol 43:783-789.

Higgs R, Cormican P, Cahalane S, Allan B, Lloyd AT, Meade K, James T, Lynn DJ, Babiuk LA and O'Farrelly C (2006) Induction of a novel chicken Toll-like receptor following Salmonella enterica serovar Typhimurium infection. Infect Immun 74:1692-1698.

Hong YH, Lillehoj HS, Lee SH, Park DW and Lillehoj EP (2006) Molecular cloning and characterization of chicken lipopolysaccharide-induced TNF-alpha factor (LITAF). Dev Comp Immunol 30:919-929.

Hu WY, Myers CP, Kilzer JM, Pfaff SL and Bushman FD (2002) Inhibition of retroviral pathogenesis by RNA interference. Curr Biol 12:1301-1311.

Janssens S and Beyaert R (2002) A universal role for MyD88 in TLR/IL-1R-mediated signaling. Trends Biochem Sci 27:474-482.

Kaiser P, Rothwell L, Avery S and Balu S (2004) Evolution of the interleukins. Dev Comp Immunol 28:375-394.

Keestra AM and van Putten JP (2008) Unique properties of the chicken TLR4/MD-2 complex: Selective lipopolysaccharide activation of the MyD88-dependent pathway. J Immunol 181:4354-4362.

Kogut MH, Genovese KJ, He HQ and Kaiser P (2008) Flagellin and lipopolysaccharide up-regulation of IL-6 and CXCLi2 gene expression in chicken heterophils is mediated by ERK1/2-dependent activation of AP-1 and NF-kB signaling pathways. Innate Immun 14:213-222.

Kudo T and Sutou S (2005) Usage of putative chicken U6 promoters for vector-based RNA interference. J Reprod Dev 51:411-417.

Kurreck J (2006) siRNA efficiency: Structure or sequence - That is the question. J Biomed Biotechnol 2006:83757.

Lavric M, Maughan MN, Bliss TW, Dohms JE, Bencina D, Keeler Jr CL and Narat M (2008) Gene expression modulation in chicken macrophages exposed to Mycoplasma synoviae or Escherichia coli. Vet Microbiol 126:111-121.

Lee JK, Kim SH, Lewis EC, Azam T, Reznikov LL and Dinarello CA (2004) Differences in signaling pathways by IL-1 beta and IL-18. Proc Natl Acad Sci USA 101:8815-8820.

Lernbecher T, Muller U and Wirth T (1993) Distinct Nf-Kappa-B Rel transcription factors are responsible for tissue-specific and inducible gene activation. Nature 365:767-770.

Leveque G, Forgetta V, Morroll S, Smith AL, Bumstead N, Barrow P, Loredo-Osti JC, Morgan K and Malo D (2003) Allelic variation in TLR4 is linked to susceptibility to Salmonella enterica serovar Typhimurium infection in chickens. Infect Immun 71:1116-1124.

Lynn DJ, Lloyd AT and O'Farrelly C (2003) In silico identification of components of the Toll-like receptor (TLR) signaling pathway in clustered chicken expressed sequence tags (ESTs). Vet Immunol Immunopathol 93:177-184.
Moynagh PN (2005) TLR signalling and activation of IRFs: Revisiting old friends from the NF-kappa B pathway. Trends Immunol 26:469-476.

O'Neill LAJ (2003) The role of MyD88-like adapters in Toll-like receptor signal transduction. Biochem Soc Trans 31:643647.

Ohl ME and Miller SI (2001) Salmonella: A model for bacterial pathogenesis. Annu Rev Med 52:259-274.

Qiu Y, Shen Y, Li X, Ding C and Ma Z (2008) Molecular cloning and functional characterization of a novel isoform of chicken myeloid differentiation factor 88 (MyD88). Dev Comp Immunol 32:1522-1530.

Roth B (1986) Design of dihydrofolate-reductase inhibitors from X-ray crystal ctructures. FASEB J 45:2765-72.

Sadeyen JR, Trotereau J, Velge P, Marly J, Beaumont C, Barrow PA, Bumstead N and Lalmanach AC (2004) Salmonella carrier state in chicken: Comparison of expression of immune response genes between susceptible and resistant animals. Microbes Infect 6:1278-1286.

Sato F, Kurokawa M, Yamauchi N and Hattori M (2006) Gene silencing of myostatin in differentiation of chicken embryonic myoblasts by small interfering RNA. Am J Physiol Cell Physiol 291:C538-C545.

Stark GR, Kerr IM, Williams BRG, Silverman RH and Schreiber RD (1998) How cells respond to interferons. Annu Rev Biochem 67:227-264.

Swaggerty CL, Kaiser P, Rothwell L, Pevzner IY and Kogut MH (2006) Heterophil cytokine mRNA profiles from genetically distinct lines of chickens with differential heterophil-mediated innate immune responses. Avian Pathol 35:102-108.

Takeda K and Akira S (2004) TLR signaling pathways. Semin Immunol 16:3-9.

Takimoto T, Takahashi K, Sato K and Akiba Y (2005) Molecular cloning and functional characterizations of chicken TL1A. Dev Comp Immunol 29:895-905.

Tamai R, Sugawara S, Takeuchi O, Akira S and Takada H (2003) Synergistic effects of lipopolysaccharide and interferongamma in inducing interleukin- 8 production in human monocytic THP-1 cells is accompanied by up-regulation of CD14, Toll-like receptor 4, MD-2 and MyD88 expression. J Endotoxin Res 9:145-153.

Tilquin P, Barrow PA, Marly J, Pitel F, Plisson-Petit F, Velge P, Vignal A, Baret PV, Bumstead N and Beaumont C (2005) A genome scan for quantitative trait loci affecting the Salmonella carrier-state in the chicken. Genet Sel Evol 37:539561.

Tong X, Yin L, Washington R, Rosenberg DW and Giardina C (2004) The p50-p50 NF-kappa B complex as a stimulusspecific repressor of gene activation. Mol Cell Biochem 265:171-183.

Underhill DM and Ozinsky A (2002) Phagocytosis of microbes: Complexity in action. Annu Rev Immunol 20:825-852.

Van LP (1996) Transcriptional activation of the chicken lysozyme gene by NF-kappa Bp65 (RelA) and c-Rel, but not by NFkappa Bp50. Biochem J 313:39-44.

Wigley P (2004) Genetic resistance to Salmonella infection in domestic animals. Res Vet Sci 76:165-169.

Wigley P, Hulme S, Rothwell L, Bumstead N, Kaiser P and Barrow P (2006) Macrophages isolated from chickens genetically resistant or susceptible to systemic salmonellosis show magnitudinal and temporal differential expression of cyto- 
kines and chemokines following Salmonella enterica challenge. Infect Immun 74:1425-1430.

Wigley P, Hulme SD, Bumstead N and Barrow PA (2002) In vivo and in vitro studies of genetic resistance to systemic salmonellosis in the chicken encoded by the SAL1 locus. Microbes Infect 4:1111-1120.

Xie H, Raybourne RB, Babu US, Lillehoj HS and Heckert RA (2003) CpG-induced immunomodulation and intracellular bacterial killing in a chicken macrophage cell line. Dev Comp Immunol 27:823-834.
Yamamoto M, Takeda K and Akira S (2004) TIR domain-containing adaptors define the specificity of TLR signaling. Mol Immunol 40:861-868.

Zhang S, Lillehoj HS, Kim CH, Keeler Jr. CL, Babu U and Zhang MZ (2008) Transcriptional response of chicken macrophages to Salmonella enterica serovar enteritidis infection. Dev Biol 132:141-151.

Associate Editor: Luiz Lehmann Coutinho

License information: This is an open-access article distributed under the terms of the Creative Commons Attribution License, which permits unrestricted use, distribution, and reproduction in any medium, provided the original work is properly cited. 